\title{
Is a Nasopharyngeal Tube Effective as Interface to Provide Bi-Level Noninvasive Ventilation?
}

\author{
Eneritz Velasco Arnaiz MD, Francisco José Cambra Lasaosa PhD, \\ Lluïsa Hernández Platero MD, Núria Millán García del Real MD, \\ and Martí Pons-Òdena MD PhD
}

\begin{abstract}
BACKGROUND: The nasopharyngeal tube (NT) is a potential interface for noninvasive ventilation (NIV) available in all health care centers. The aim of the study was to describe our experience in the use of the NT for bi-level NIV in infants and its effectiveness. METHODS: Prospective observational study from January 2007 to December 2010, including all patients $\leq 6$ months old admitted to the pediatric ICU (PICU) and treated with NIV with two levels of pressure using the NT. Clinical data collected before starting NIV, and at $2,8,12$ and $24 \mathrm{~h}$, were analyzed following NIV initiation: first-line or initial NIV (i-NIV), elective postextubation NIV (e-NIV), and rescue postextubation NIV (r-NIV). The need for intubation was considered to be NIV failure. RESULTS: One hundred fifty-one episodes of NIV were included in the study, with $65 \%$ of patients having bronchiolitis. e-NIV was most frequently used (48\%) (i-NIV $44 \%$, r-NIV $8 \%$ ), and the failure rate, $27 \%$ in total, was highest in the i-NIV group $(37 \%)$ (e-NIV $18 \%$, r-NIV $25 \%)$. Case patients with successful outcomes had shorter PICU stays $(8.5$ vs $13 \mathrm{~d}, P=.001)$ and hospital stays $(17$ vs $23 \mathrm{~d}, P=.03)$ stays. The NT needed to be changed for another interface in only 5 case patients, few complications (4 of 151 patients) were observed, and mortality ( 2 of 151 patients) was unrelated to NIV. CONCLUSIONS: Use of the NT showed $73 \%$ effectiveness, with few complications. The effectiveness was higher in e-NIV than i-NIV. Key words: airway extubation; bronchiolitis; infant; noninvasive ventilation; pediatric intensive care units; respiratory insufficiency. [Respir Care 2014;59(4):510-517.

(C) 2014 Daedalus Enterprises]
\end{abstract}

\section{Introduction}

Using noninvasive ventilation (NIV) as a treatment for respiratory failure requires the availability of appropriate material and well-trained personnel.

Interface selection is fundamental to optimize the interaction between patient and ventilator. ${ }^{1}$ When nasal prongs

All authors are affiliated with the Pediatric Intensive Care Unit, Hospital Universitario Sant Joan de Déu, Barcelona, Spain.

This study was funded, in part, by Invest for Children Foundation, who donated 3 NIV ventilators and provided funding for a data manager. Dr Pons-Òdena has been a speaker for Maquet, receiving honoraria on one occasion. The other authors have disclosed no conflicts of interest.

Correspondence: Martí Pons-Òdena MD, Pediatric Intensive Care Unit, Hospital Universitario Sant Joan de Déu, Passeig de Sant Joan de Déu 2, 08950 Esplugues de Llobregat, Barcelona. E-mail: mpons@ hsjdbcn.org.

DOI: $10.4187 /$ respcare. 02556 cannot be used in small infants, it is occasionally necessary to adapt some interfaces designed for older patients, like the nasal interface used as oronasal, ${ }^{2-4}$ as there are few commercially-available interfaces which fit well in this age group. The nasopharyngeal tube (NT) or single long nasal prong, which has been in use since the 1970 s, could be another alternative. ${ }^{5}$ Although double nasal prongs have been shown to be more effective than single nasal devices for CPAP in premature babies, ${ }^{6}$ no studies to date have analyzed the results with the NT in the pediatric population.

From 1998 to 2006, all the infants in our pediatric ICU (PICU) failing with CPAP were intubated without a previous trial with bi-level pressure, mainly due to the lack of appropriate ventilators and interfaces. The present study aims to describe our experience in introducing the use of the NT as a single nasal interface for NIV with 2 levels of pressure in infants up to 6 months of age in a PICU and to analyze its effectiveness. 


\section{Nasopharyngeal Tube as an Interface for Bi-LeVel NIV}

\section{Methods}

A prospective observational study was carried out in the PICU of a tertiary-care hospital with 14 beds for critical patients ranging in age from 0 to $18 \mathrm{y}$, from January 2007 to December 2010.

\section{Subjects}

All patients $\leq 6$ months old who were treated with bilevel pressure NIV using the NT were included in the study. Approval from the ethics board of the Hospital Universitario Sant Joan de Déu was obtained for collecting NIV data. As NIV is a routine treatment in the PICU, specific informed consent was not considered necessary.

\section{NIV Strategy}

A shortened endotracheal tube inserted through a naris and secured in a way where $7 \mathrm{~cm}$ were introduced into the hypopharynx was used as the interface. Because almost $75 \%$ of the patients were $<3$ months old, the length of the NT used was $7 \mathrm{~cm}$.

Ventilator selection was based on patient age, ventilator availability, our previous experience, and the published data on the trigger sensitivity of each of the following devices: Giulia (Ginevri, Rome, Italy) was used for infants $<1$ month old ${ }^{7}$; Servo-i (Maquet, Rastatt, Germany) for infants between 1 and 3 months old ${ }^{4}$; and BiPAP Vision (Respironics, Andover, Massachusetts) and Carina (Dräger, Lübeck, Germany) for those $>3$ months old. A heated humidifier (Fisher \& Paykel Healthcare, Auckland, New Zealand) was used in all cases.

The ventilation strategy was applied in accordance with the protocol of the Respiratory Group of the Spanish Society of Pediatric Intensive Care. ${ }^{8}$ Starting positive inspiratory pressure (peak inspiratory pressure or inspiratory positive airway pressure [IPAP]) was set between 6 and 8 $\mathrm{cm}_{2} \mathrm{O}$, and positive end-expiratory pressure (or expiratory positive airway pressure [EPAP]) was set at $4 \mathrm{~cm}$ $\mathrm{H}_{2} \mathrm{O}$. According to the clinician's criteria, if inspiratory volume was inadequate, or work of breathing or hypercapnia did not diminish, the IPAP was increased progressively to a maximum of $22 \mathrm{~cm} \mathrm{H}_{2} \mathrm{O}$, and the EPAP was increased to a maximum of $8 \mathrm{~cm} \mathrm{H}_{2} \mathrm{O}$ to improve alveolar recruitment and oxygenation. In the cases where any signs of NIV treatment failure were observed $\left(\mathrm{S}_{\mathrm{pO}_{2}}<85 \%, \mathrm{p}_{\mathrm{CO}_{2}}\right.$ $>65 \mathrm{~mm} \mathrm{Hg}$, or an increase in the signs of respiratory difficulty [i.e., greater respiratory/heart rate or work of breathing]) despite maximum parameters of support, and whenever any exclusion criteria for $\mathrm{NIV}^{9}$ appeared, endotracheal intubation was performed and conventional mechanical ventilation was started. To overcome the presence of leaks, both inspiratory and expiratory asynchrony were

\section{QUICK LOOK}

\section{Current knowledge}

Noninvasive ventilation (NIV) is commonly applied with a variety of nasal and oro-nasal masks. In infants, the use of a nasopharyngeal tube represents an option for non-invasive ventilation.

\section{What this paper contributes to our knowledge}

The use of NIV delivered via a naso-tracheal tube in patients less than 6 months of age prevented intubation in two-thirds of patients studied. NIV via a nasotracheal tube was more successful for prevention of re-intubation than prevention of intubation during the initial NIV application.

reduced using the continuous mandatory ventilation pressure mode with a fixed inspiratory time. ${ }^{10}$

In patients where nonpharmacologic methods were insufficient to favor patient adaptation to the interface, the following sedatives were used, alone or in combination, depending on the physician's criteria: oral levomepromazine $(1 \mathrm{mg} / \mathrm{kg} / \mathrm{dose})$ and, more commonly for patients being weaned from invasive ventilation, intravenous midazolam $(0.05-0.1 \mathrm{mg} / \mathrm{kg} / \mathrm{h})$ were maintained or switched to propofol (1-2 $\mathrm{mg} / \mathrm{kg} / \mathrm{h}$ ) for short periods of time.

A nasogastric tube was placed to avoid gastric distention and vomiting during the course of NIV, and for feeding when the clinical situation permitted.

All patients were continuously monitored for heart rate, breathing frequency, and $\mathrm{S}_{\mathrm{pO}_{2}}$. Blood gas levels were obtained by the physician when deemed necessary.

\section{Variables}

Patients' age, gender, and weight were documented for each episode, as was the Pediatric Risk of Mortality Score II in the first $24 \mathrm{~h}$ after PICU admission, ${ }^{11}$ the presence of underlying conditions, the days and parameters of conventional mechanical ventilation in previously intubated patients, and the following clinical variables of NIV support: the pathology that led to respiratory failure; type of ventilator and ventilation mode; ventilation parameters $\left(\mathrm{F}_{\mathrm{IO}_{2}}\right.$, IPAP, and EPAP); and physiological variables (heart rate, breathing frequency, $\mathrm{S}_{\mathrm{pO}_{2}}$, and $\mathrm{S}_{\mathrm{pO}_{2}} / \mathrm{F}_{\mathrm{IO}_{2}}[\mathrm{SF}]$ ratio) prior to starting NIV at $2,8,12$, and $24 \mathrm{~h}$; blood gas measurements ( $\mathrm{pH}, \mathrm{P}_{\mathrm{O}_{2}}, \mathrm{P}_{\mathrm{CO}_{2}}$, and bicarbonate) according to the physician's criteria; need to change ventilation mode, ventilator, and/or interface; use of pharmacologic sedation; appearance of complications or contraindications; mortality; NIV duration; success/failure of NIV; and duration of PICU and hospital stays. 


\section{Nasopharyngeal Tube as an Interface for Bi-LeVel NIV}

To calculate the SF ratio, $\mathrm{S}_{\mathrm{pO}_{2}}$ values $>97 \%$ were excluded, and SF ratio values were correlated to $\mathrm{P}_{\mathrm{aO}_{2}}\left(\mathrm{P}_{\mathrm{O}_{2}}\right.$ in arterial blood $) / \mathrm{F}_{\mathrm{IO}_{2}}$ ratio values in accordance with the results published by Khemani et al. ${ }^{12}$

The patient population was subgrouped according to the NIV indication:

1. First-line or initial NIV (i-NIV) without previous conventional mechanical ventilation: in the initial phase of respiratory failure, after the failure of a CPAP trial in the cases where CPAP was indicated (apneas and type 1 respiratory failure). In the study period, CPAP was provided in most cases using the Benveniste device with a NT. This device does not allow ongoing pressure monitoring and has an effectiveness of 54\%

2. Elective postextubation NIV (e-NIV): immediately after extubation due to previous extubation failure or not meeting the standard extubation criteria

3. Rescue NIV (r-NIV): after extubation, due to the appearance of respiratory failure; NIV was considered successful when the patient avoided endotracheal intubation. Episodes where NIV was withdrawn due to the appearance of contraindications were excluded from failure analysis.

\section{Statistical Analysis}

Analysis was carried out with the statistics program SPSS version 18.0 (IBM, New York, New York). Descriptive statistics are shown through proportions with $95 \%$ CIs for categorical variables; with means and medians as measures of central tendency; and SD and interquartile range as measures of dispersion for quantitative variables. In view of the limitations arising from the small sample size of the r-NIV group and the diverse character of the e-NIV group, which complicate the analysis of postextubation NIV as a whole, we decided, a posteriori, to exclude r-NIV episodes from statistical hypothesis tests, which were performed only in the i-NIV and e-NIV groups. NIV outcome was correlated to the distinct variables analyzed, using the chi-square test, the Student $t$ test, and Mann-Whitney U test accordingly. Multivariate analysis was performed with the Cox regression model. Statistical significance was established as a $P$ value of $<.05$.

Prior data indicate that the failure rate among control subjects is $0.24 .{ }^{13}$ If the true failure rate for experimental subjects were 0.4 , we would need to study 144 experimental subjects to reject the null hypothesis that the failure rates for experimental and control subjects are equal with a $P$ value (power) of 0.8 . The type 1 error probability associated with the test of this null hypothesis is 0.05 . We used a continuity-corrected chi-square statistic or Fisher exact test to evaluate this null hypothesis.

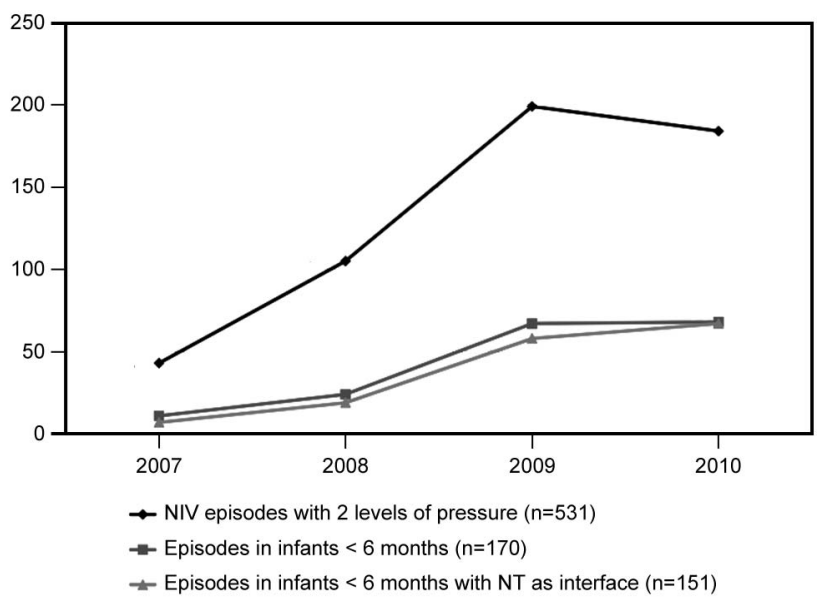

Fig. 1. NIV episodes where nasopharyngeal tube was in use.

\section{Results}

Of 170 hospital admissions of infants $<6$ months old who received NIV treatment with 2 levels of pressure during the study period, the NT was used in $151(89 \%)$. The yearly distribution of the episodes is shown in Figure 1. Twenty five percent of infants had been treated previously with CPAP.

The most frequent causes for the use of NIV were bronchiolitis (98 of 151 patients; 65\%), cardiac surgery (14 of 151 patients; $9.5 \%$ ), and other respiratory infections (13 of 151 patients; $8.5 \%$ ) such as pertussis (3 of 13 patients; $23 \%)$. NIV was used on 66 of 151 occasions (44\%) as i-NIV, and on 85 of 151 occasions (56\%) after extubation, 72 of $85(85 \%)$ as e-NIV and 13 of $85(15 \%)$ as r-NIV. The baseline characteristics of the episodes are summarized in Table 1.

The most frequently used ventilator was the Servo-i device (136 of 151 occasions; 90\%), and the most frequently used mode was continuous mandatory ventilation pressure (113 of 151 occasions; $75 \%$ ). In 58 of 151 episodes (38\%), the mode was changed: in 43 of 58 occasions (74\%), mode was changed to CPAP as a step preceding the withdrawal of ventilatory support. In 28 of these 58 episodes, the ventilator was also changed, most commonly to the Giulia device (13 of 28 episodes; $46 \%$ ) and the Benveniste device (10 of 28 episodes; 36\%). An interface change was only necessary in 5 of 151 cases (3\%), one to binasal prongs and the rest to an oronasal interface.

In 100 episodes (66\%), some type of sedative was used, alone (85 of 100 episodes; $85 \%$ ) or in combination (15 of 100 episodes; $15 \%$ ). The most commonly used medication was oral levomepromazine (64 of 100 episodes; 64\%), followed by propofol in a continuous drip (32 of 100 episodes; $32 \%$ ) for short periods, and midazolam (19 of 100 episodes; 19\%). 


\section{Nasopharyngeal Tube as an Interface for Bi-Level NIV}

Table 1. Baseline Characteristics of the Episodes at NIV Initiation

\begin{tabular}{|c|c|c|c|c|}
\hline Characteristics & i-NIV $(n=66)$ & e-NIV $(n=72)$ & $P^{*}$ & r-NIV $(n=13)$ \\
\hline Age, months & $1.6(0.8-2.9)$ & $1.4(0.8-3.1)$ & & $2.4(1.2-4.3)$ \\
\hline \multicolumn{5}{|l|}{ Sex } \\
\hline Female & $27(41)$ & $25(35)$ & & $4(31)$ \\
\hline Male & $39(59)$ & $47(65)$ & & $9(69)$ \\
\hline Weight, kg & $4.4(3.4-5.3)$ & $4.9(3.3-5.7)$ & & $3.4(3.2-4.6)$ \\
\hline PRISM II score & $4(0-8.7)$ & $9(4-9.5)$ & $0.03 \dagger$ & $9(7-9)$ \\
\hline Underlying condition & $22(33)$ & $31(43)$ & & $9(69)$ \\
\hline Prematurity & $9(41)$ & $11(35)$ & & $2(22)$ \\
\hline Cardiopathy & $5(23)$ & $10(32)$ & & $6(67)$ \\
\hline Down syndrome & $1(4)$ & $3(10)$ & & \\
\hline Neuromuscular & $2(9)$ & $3(10)$ & & \\
\hline Oncological & & $1(3)$ & & \\
\hline Others & $5(23)$ & $3(10)$ & & $1(11)$ \\
\hline \multicolumn{5}{|l|}{ Admitting pathology } \\
\hline Bronchiolitis & $51(77)$ & $44(61)$ & $0.02 \ddagger$ & $5(38)$ \\
\hline Cardiac postoperative & & $9(13)$ & 0.001 \$ & $4(31)$ \\
\hline Respiratory infections & $6(9)$ & $7(10)$ & & \\
\hline Sepsis & & $1(1)$ & & \\
\hline Acute pulmonary edema & $3(5)$ & $1(1)$ & & $2(15)$ \\
\hline Bronchospasm/wheezing & $2(3)$ & $2(3)$ & & $1(8)$ \\
\hline Apnea & $3(5)$ & $3(5)$ & & \\
\hline Atelectasis & $1(1)$ & $2(3)$ & & $1(8)$ \\
\hline Bronchoaspiration & & $1(1)$ & & \\
\hline Myopathy with insufficient respiratory effort & & $1(1)$ & & \\
\hline Postextubation croup & & $1(1)$ & & \\
\hline \multicolumn{5}{|l|}{ Vital signs } \\
\hline $\mathrm{f}$, breaths/min & $42(33-51)$ & $32(28-36)$ & $0.001 \dagger$ & $30(25-36)$ \\
\hline HR, beats/min & $167 \pm 23$ & $142 \pm 24$ & $0.001 \S$ & $147 \pm 26$ \\
\hline $\mathrm{S}_{\mathrm{pO}_{2}}, \%$ & $98(94-100)$ & $96(94-98)$ & & $99(97-100)$ \\
\hline \multicolumn{5}{|l|}{ Support prior to NIV } \\
\hline Intubation, days & & $7(4-10)$ & & $2(1-5.5)$ \\
\hline $\mathrm{F}_{\mathrm{IO}_{2}}, \%$ & $50(30-61)$ & $30(27-35)$ & $0.001 \dagger$ & $35(30-50)$ \\
\hline Positive inspiratory pressure, $\mathrm{cm} \mathrm{H}_{2} \mathrm{O}$ & & $16(14-22)$ & & $17(12-21)$ \\
\hline PEEP, $\mathrm{cm} \mathrm{H}_{2} \mathrm{O}$ & & $5(5-6)$ & & $5(4.5-6.5)$ \\
\hline SF ratio & $234(147-330)(n=20)$ & $310(269-323)(n=47)$ & $0.02 \dagger$ & $277(194-277)(n=3)$ \\
\hline \multicolumn{5}{|l|}{ Blood gas measurements } \\
\hline $\mathrm{p}_{\mathrm{aO}_{2}}, \mathrm{~mm} \mathrm{Hg}$ & $89(64-143)(n=10)$ & $67(59-108)(n=5)$ & & $116(114-116)(n=3)$ \\
\hline $\mathrm{pH}$ & $7.38(7.26-7.42)(n=17)$ & $7.40(7.37-7.42)(n=17)$ & & $7.32(7.19 ; 7.41)(n=4)$ \\
\hline $\mathrm{p}_{\mathrm{CO}_{2}}, \mathrm{~mm} \mathrm{Hg}$ & $42(37-66)(n=18)$ & $45(38-51)(n=16)$ & & $50(36-68)(n=4)$ \\
\hline Bicarbonate, $\mathrm{mmol} / \mathrm{L}$ & $25(24-26)(n=15)$ & $26(23-29)(n=16)$ & & $23(20-24)(n=4)$ \\
\hline $\begin{array}{l}\text { Data are given as median (interquartile range), } n \text { (\%), or mean } \\
\text { * Variables for which statistically significant differences betwee } \\
\uparrow \text { Mann-Whitney U test for the analysis of quantitative variable } \\
\text { † Chi-square test was used for the analysis of categorical variab } \\
\S \text { Student } t \text { test for the analysis of quantitative variables. } \\
\text { PRISM = Pediatric Risk of Mortality } \\
\mathrm{f}=\text { breathing frequency } \\
\mathrm{HR}=\text { heart rate } \\
\mathrm{i} \text {-NIV = initial noninvasive ventilation } \\
\text { e-NIV = elective postextubation noninvasive ventilation } \\
\text { r-NIV = rescue postextubation noninvasive ventilation } \\
\mathrm{SF} \text { ratio }=\mathrm{S}_{\mathrm{pO}_{2}} / \mathrm{F}_{\mathrm{IO}_{2}} \text { ratio }\end{array}$ & $\begin{array}{l}\text { D. } \\
\text { NIV and e-NIV were obtained are ind }\end{array}$ & & & \\
\hline
\end{tabular}

Complications were observed in 4 of 151 cases (3\%), 3 in the e-NIV group and 1 in the i-NIV group. One of them, a case of bronchoaspiration, caused the failure of NIV in a 5-month-old baby girl with meningococcal sepsis who 


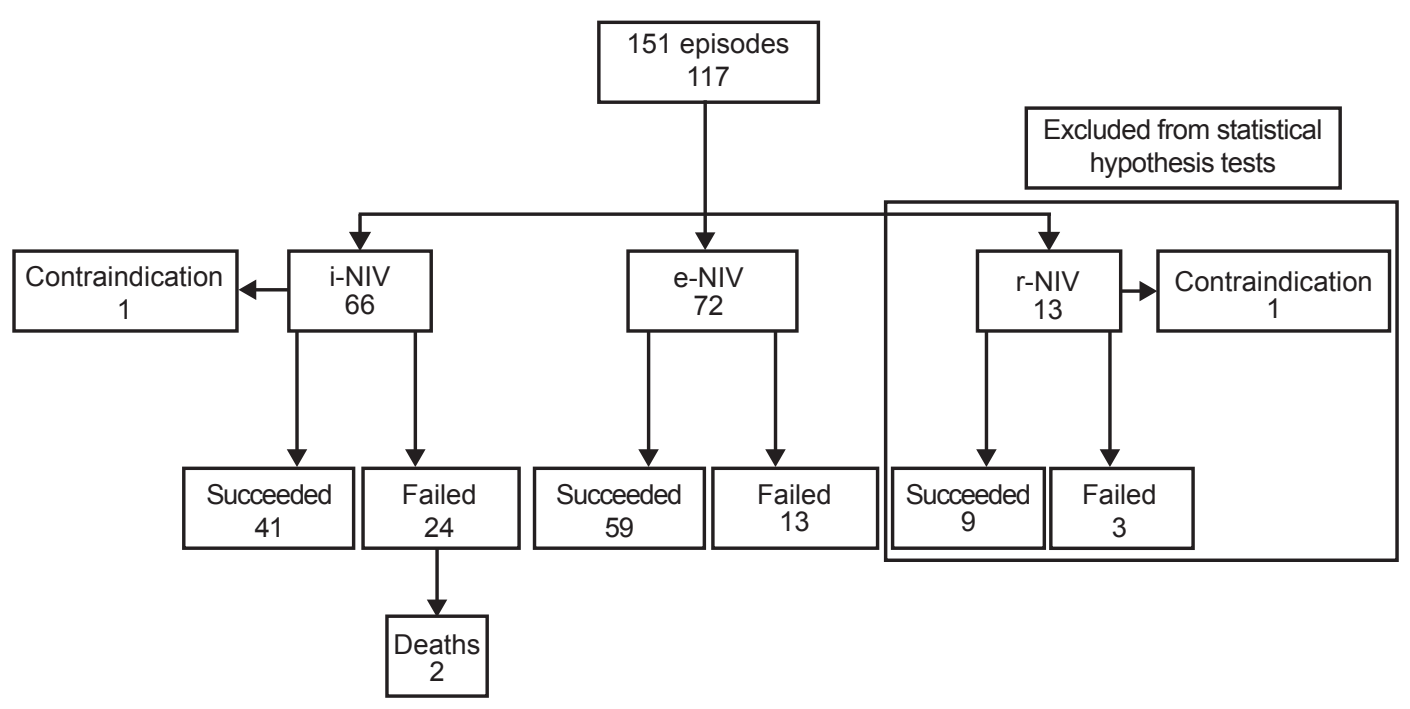

Fig. 2. NIV outcomes flowchart.

experienced clinical deterioration on the third day of eNIV due to a nosocomial infection. The remaining episodes were interface obstruction due to secretions.

In 2 of 151 cases (1\%), NIV was suspended due to the need for intubation for surgery.

Two patients died from conditions unrelated to NIV: a newborn girl admitted to the hospital at 11 days of life for bronchiolitis, who was intubated within 1 hour of NIV initiation, and after receiving extracorporeal membrane oxygenation for 14 days; and a 3-month-old girl with decompensated, complex congenital heart disease a week after intubation following i-NIV due to progression of hepatic failure.

\section{Analysis of NIV efficacy}

The distribution of the included episodes and NIV outcomes are summarized in Figure 2.

The success rate was 109 of 149 cases (73\%), with no differences based on the year data were collected, presence of underlying conditions, Pediatric Risk of Mortality Score II score, pathology that caused the respiratory failure, type of ventilator and ventilation mode, or the use of sedatives. In the cases where NIV failed (i-NIV, e-NIV, and r-NIV), the duration of support was shorter $(12 \mathrm{~h}$ [interquartile range $2.2-28 \mathrm{~h}$ ] vs $75.5 \mathrm{~h}$ [interquartile range $46.5-113.5 \mathrm{~h}$ ]; $P$ $=.001)$, and the PICU and hospital stays were longer than in the successful cases (13 days [interquartile range 9-23.5 days] vs 8.5 days [interquartile range $5-14$ days], $P=.001$; and 23 days [interquartile range 16-37 days] vs 17 days [interquartile range 12-30.5 days], $P=.03$, respectively).

The rates of failure and the duration of ventilatory support and hospital admission are shown in Table 2. In Table 3 , the variables for which statistically significant differ- ences were observed between episodes where NIV was successful or failed in each group are detailed.

In the Cox regression analysis, decrease in heart rate and breathing frequency at $2 \mathrm{~h}$, and IPAP value at $12 \mathrm{~h}$, were entered, and the analysis was adjusted to the type of NIV used (i-NIV, e-NIV, or r-NIV), showing IPAP at $12 \mathrm{~h}$ (hazard ratio $0.980,95 \% \mathrm{CI} 0.961-0.999 ; P=.04$ ) and decrease in heart rate at $2 \mathrm{~h}$ (hazard ratio $1.298,95 \% \mathrm{CI}$ $1.046-1.611 ; P=.02)$ as independent predictive factors for NIV failure.

\section{Discussion}

Few studies have analyzed the effectiveness of interfaces in pediatrics, ${ }^{14-16}$ and, to our knowledge, this is the first one focused on the NT for bi-level NIV in infants. The observational design of the study and the fact that it was performed over a short period of time, in a single center, without variation in protocol or materials used, and without a control group, confer homogeneity to our sample but may limit the generalization of some of the results obtained.

The lack of evidence in favor of one or another interface, the availability of material, and the practical experience of the PICU staff conditioned the use of the NT in the large majority of case patients in this age range during the study period. With the current protocol, the success rate, nearly $75 \%$ of the episodes included, was similar to those of other studies about pediatric NIV,,$^{2-4,13,17-19}$ few complications were documented, and the mortality observed was not attributable to NIV per se.

The separate analyses of i-NIV, e-NIV, and r-NIV is common in adult studies as the baseline characteristics, responses, and outcomes are different in each group. In 


\section{Nasopharyngeal Tube as an Interface for Bi-Level NIV}

Table 2. NIV Failure rate, Duration of NIV, and Admissions at NIV Initiation

\begin{tabular}{|c|c|c|c|c|}
\hline Characteristics & i-NIV $(n=65)^{*}$ & e-NIV $(n=72)$ & $P \dagger$ & $\mathrm{r}-\mathrm{NIV}(n=12)$ \\
\hline Failure & $24(37)$ & $13(18)$ & .01 & $3(25)$ \\
\hline \multicolumn{5}{|l|}{ NIV } \\
\hline Duration, $\mathrm{h}$ & $61(12-93)$ & $58(33-96)$ & & $34(16-76)$ \\
\hline Success & $82(63-116)$ & $68(39-102)$ & & $23(14-86)$ \\
\hline Failure & $8(2-17)$ & $28(10-60)$ & .01 & $38(30-38)$ \\
\hline \multicolumn{5}{|l|}{ Stay, days } \\
\hline PICU & $7(4-10)$ & $14(9-22.7)$ & .001 & $10(4.7-12.7)$ \\
\hline Success & $5(4-8)$ & $11(8-19)$ & & $10(3.5-12)$ \\
\hline Failure & $10(8.2-13.7)$ & $29(18.5-68)$ & .001 & $12(7-12)$ \\
\hline Hospital & $16(10.5-24)$ & $24(15-37)$ & .001 & $15(12.2-22.2)$ \\
\hline Success & $13(9-24.5)$ & $18(14-35)$ & & $15(10-19.5)$ \\
\hline Failure & $17(14.2-23.7)$ & $37(27-101)$ & .001 & $20(12-20)$ \\
\hline $\begin{array}{l}\text { Data are given as } n \\
* \text { One subject was ex } \\
\dagger \text { Statistically signific } \\
\text { PICU = pediatric IC } \\
\text { NIV = noninvasive } \\
\text { i-NIV = initial nonin } \\
\text { e-NIV = elective pos } \\
\text { r-NIV = rescue poste }\end{array}$ & $\begin{array}{l}\text { tile range), unless otherw } \\
\text { cause a contraindication a } \\
\text { i-NIV and e-NIV. Chi-sq } \\
\text { ventilation } \\
\text { entilation }\end{array}$ & e analysis of categorical & itney U & antitative variables. \\
\hline
\end{tabular}

our study, the small sample size of the r-NIV group hindered comparisons with the other two groups. Nevertheless, without trying to draw conclusions, we have shown the descriptive analysis of the r-NIV group where the success rate was similar to what has been described in the literature. Similar to other studies, ${ }^{20}$ e-NIV was more effective than i-NIV, with half the number of failures. This observation is probably related to the difference in the severity of respiratory distress at the time NIV was started, with significantly lower breathing frequency, heart rate, and $\mathrm{F}_{\mathrm{IO}_{2}}$ requirements, and a significantly higher SF ratio in the e-NIV group at baseline. Having received invasive ventilation first could lead to a faster recovery from the acute phase in the e-NIV group and, consequently, to a higher NIV success rate.

In both the i-NIV and e-NIV groups, the main cause of failure was considered to be the progression of the illness that had caused the PICU admission. Sedatives were required more frequently than in previous reports, which predominantly used CPAP (Mayordomo et al ${ }^{21}$ described a sedation rate of $\sim 45 \%$ ). But, as previously demonstrated by Essouri et al, ${ }^{22}$ leaks are not as influential on asynchrony in patients receiving CPAP as they are in patients receiving bi-level pressure. However, although intolerance to the interface and/or adaptation problems with NIV were documented, asynchrony was not quantified, and this could have influenced some of the failures.

Unfortunately, the comfort level of our patients was not monitored, not unlike previous reports. The need for pharmacologic sedation in up to two thirds of episodes may reflect the inherent difficulty in the adaptation and syn- chronization of this age group to bi-level support, but it can also be pointed out that $56 \%$ of our patients had been intubated previously, so the sedatives were still being withdrawn. Regarding the use of propofol in almost one third of the episodes where sedatives were administered, we did not observe any associated adverse events. However, even though propofol can be used for short periods in pediatric patients, we are not promoting it as a first-line drug in all cases, and other alternatives may be considered.

In the i-NIV group, sustained hypoxemia with a greater $\mathrm{F}_{\mathrm{IO}_{2}}$ requirement, a lower SF ratio at 12 and $24 \mathrm{~h}$, and a greater increase of heart rate at $12 \mathrm{~h}$ were associated with a higher failure rate.

In the e-NIV group, failure was higher in girls, a fact for which we have no hypothesis; in patients who received more sedation, possibly due to greater adaptation problems after sedation was decreased during the weaning process of conventional mechanical ventilation; and in the cases with a greater increase of breathing frequency at $2 \mathrm{~h}$.

Multivariate analysis, as in other studies, indicated that a smaller decrease in heart rate is the most important predictive factor for failure. . $^{3,4} \mathrm{~A}$ mean airway pressure value $>11 \mathrm{~cm} \mathrm{H} \mathrm{O}_{2} \mathrm{O}^{17}$ was also identified in a study using conventional ventilators. As this value is not measurable in NIV-specific ventilators, we found that a higher IPAP value at $12 \mathrm{~h}$ was a predictive factor for failure in our sample.

As a whole, the success of NIV in the episodes included in the study, with $<5$ days of maximum support, meant a shorter PICU length of stay. The decrease in hospital stay and the rates of intubation/re-intubation and conventional mechanical ventilation could influence the associated mor- 


\section{Nasopharyngeal Tube as an Interface for Bi-Level NIV}

Table 3. Differences Between Successful and Failed Episodes of i-NIV and e-NIV

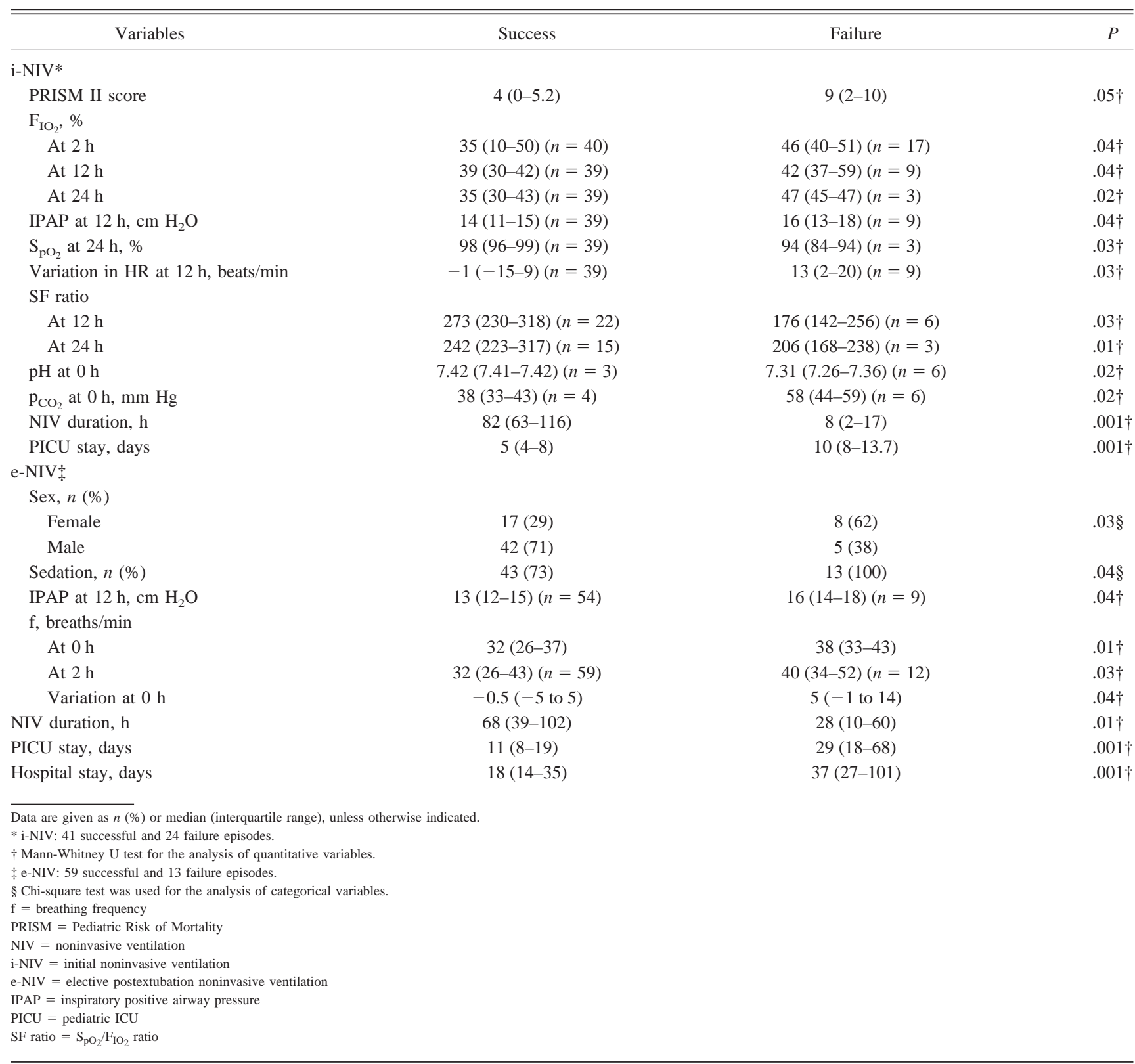

bidity and mortality, making it crucial to develop properly designed studies to determine the predictive factors for success and failure that would contribute to a better selection of patients who would benefit from starting and maintaining NIV.

Although the ideal interface does not exist, selecting an inappropriate one could contribute to NIV failure. Therefore, besides developing and commercializing appropriate interfaces for diverse ages and situations, it is essential to know the advantages and disadvantages of the ones that are currently available. The NT is a cheap and easily available interface, which could be obstructed by secretions. It also has a predictable presence of leaks as a limiting factor for synchronization, especially if a pressure support mode is selected. Despite all this, we believe that, due to the continuous care and respiratory hygiene measures, tube change was required on very few occasions, complications were rare, and pressure sores from interfaces frequently described in the literature $3,4,13,18,23,24$ were not observed.

We would like to stress that clinicians should be cautious about using the NT interface with ventilators other than Servo-i or BiPAP Vision. Their superiority in compensating for leaks has been shown in a bench study, ${ }^{25}$ so it must be pointed out that different ventilators may offer different results. 


\section{Nasopharyngeal Tube as an Interface for Bi-LeVel NIV}

\section{Conclusions}

In infants failing with CPAP or receiving bi-level pressure as a first step according to the Respiratory Group of the Spanish Society of Pediatric Intensive Care protocol, ${ }^{8}$ the NT is an alternative interface capable of providing effective NIV with two levels of pressure and with few complications in $73 \%$ of infants $\leq 6$ months old. Using the same ventilators and an appropriate protocol of care, we believe it could be applicable to other health care centers.

The effectiveness of NIV varies according to the moment it is started, being higher in the e-NIV group.

\section{REFERENCES}

1. Navalesi P, Fanfulla F, Frigerio P, Gregoretti C, Nava S. Physiologic evaluation of non-invasive mechanical ventilation delivered with three types of masks in patients with chronic hypercapnic respiratory failure. Crit Care Med 2000;28(6):1785-1790.

2. Essouri S, Chevret L, Durand P, Haas V, Fauroux B, Devictor D. Non-invasive positive pressure ventilation: five years of experience in a pediatric intensive care unit. Pediatr Crit Care Med 2006;7(4): 329-334.

3. Mayordomo-Colunga J, Medina A, Rey C, Diaz JJ, Concha A, Los Arcos M, et al. Predictive factors of non-invasive ventilation failure in critically ill children: a prospective epidemiological study. Intensive Care Med 2009;35(3):527-536.

4. Yanez LJ, Yunge M, Emilfork M, Lapadula M, Alcantara A, Fernandez $\mathrm{C}$, et al. A prospective, randomized, controlled trial of noninvasive ventilation in pediatric acute respiratory failure. Pediatr Crit Care Med 2008;9(5):484-489.

5. Boros SJ, Reynolds JW. Prolonged apnea of prematurity: Treatment with continuous airway distending pressure delivered by nasopharyngeal tube. Clin Pediatr (Phila) 1976;15(2):123-134.

6. De Paoli AG, Davis PG, Faber B, Morley CJ. Devices and pressure sources for administration of nasal continuous positive airway pressure (NCPAP) in preterm neonates. Cochrane Database Syst Rev 2008;(1):CD002977.

7. Pons-Òdena M, Lasuen N, Palomeque A. Non-invasive ventilation in infant younger than 2 months: synchronicity is the key-point. Pediatr Crit Care Med 2007;8:A357.

8. Pons-Òdena M, Cambra Lasaosa FJ, Sociedad Española de Cuidados Intensivos Pediátricos. Mechanical ventilation in pediatrics (III). Weaning, complications and other types of ventilation. Non-invasive ventilation. An Pediatr (Barc) 2003;59(2):165-172.

9. Gaboli M, Pons-Òdena M. Indications and contra-indications of noninvasive ventilation. In: Medina A, Pons-Òdena M, Martinon-Torres F, editors. Non-invasive ventilation in pediatrics, 2nd edition. Madrid: Ergon; 2009:11-16.

10. Calderini E, Confalonieri M, Puccio PG, Francavilla N, Stella L, Gregoretti C. Patient-ventilator asynchrony during non-invasive ven- tilation: the role of expiratory trigger. Intensive Care Med 1999; 25(7):662-667.

11. Pollack MM, Patel KM, Ruttimann UE. PRISM III: an updated pediatric risk of mortality score. Crit Care Med 1996;24(5):743-752.

12. Khemani RG, Patel NR, Bart RD 3rd, Newth CJ. Comparison of the pulse oximetric saturation/fraction of inspired oxygen ratio and the $\mathrm{PaO}_{2}$ /fraction of inspired oxygen ratio in children. Chest 2009;135(3): 662-668.

13. Lum LC, Abdel-Latif ME, de Bruyne JA, Nathan AM, Gan CS Noninvasive ventilation in a tertiary pediatric intensive care unit in a middle-income country. Pediatr Crit Care Med 2011;12(1):e7-e13.

14. Piastra M, Antonelli M, Chiaretti A, Polidori G, Polidori L, Conti G. Treatment of acute respiratory failure by helmet-delivered non-invasive pressure support ventilation in children with acute leukemia: a pilot study. Intensive Care Med 2004;30(3):472-476.

15. Chidini G, Calderini E, Cesana BM, Gandini C, Prandi E, Pelosi P. Non-invasive continuous positive airway pressure in acute respiratory failure: Helmet versus facial mask. Pediatrics 2010;126(2):e330e336.

16. Figueruelo AG, Villaescusa JU, Prieto MB, Garcia MJ, Bartolome SM, Cid JL. Use of high-flow nasal cannula for non-invasive ventilation in children. An Pediatr (Barc) 2011;75(3):182-187.

17. Munoz-Bonet JI, Flor-Macian EM, Brines J, Rosello-Millet PM, Cruz Llopis M, Lopez-Prats JL, et al. Predictive factors for the outcome of non-invasive ventilation in pediatric acute respiratory failure. Pediatr Crit Care Med 2010;11(6):675-680.

18. Nunes P, Abadesso C, Almeida E, Silvestre C, Loureiro H, Almeida $\mathrm{H}$. Non invasive ventilation in a pediatric intensive care unit. Acta Med Port 2010;23(3):399-404.

19. Joshi G, Tobias JD. A five-year experience with the use of BiPAP in a pediatric intensive care unit population. $\mathrm{J}$ Intensive Care Med 2007;22(1):38-43.

20. James CS, Hallewell CP, James DP, Wade A, Mok QQ. Predicting the success of non-invasive ventilation in preventing intubation and re-intubation in the paediatric intensive care unit. Intensive Care Med 2011;37(12):1994-2001.

21. Mayordomo-Colunga J, Medina A, Rey C, Los Arcos M, Concha A, Menendez S. Success and failure predictors of non-invasive ventilation in acute bronchiolitis. An Pediatr (Barc) 2009;70(1):34-39.

22. Essouri S, Nicot F, Clement A, Garabedian EN, Roger G, Lofaso F, et al. Non-invasive positive pressure ventilation in infants with upper airway obstruction: Comparison of continuous and bilevel positive pressure. Intensive Care Med 2005;31(4):574-580.

23. Bernet V, Hug MI, Frey B. Predictive factors for the success of non-invasive mask ventilation in infants and children with acute respiratory failure. Pediatr Crit Care Med 2005;6(6):660-664.

24. Munoz-Bonet JI, Flor-Macian EM, Rosello PM, Llopis MC, Lizondo A, Lopez-Prats JL, et al. Non-invasive ventilation in pediatric acute respiratory failure by means of a conventional volumetric ventilator. World J Pediatr 2010;6(4):323-330.

25. Ferreira JC, Chipman DW, Hill NS, Kacmarek RM. Bilevel vs ICU ventilators providing non-invasive ventilation: effect of system leaks: a COPD lung model comparison. Chest 2009;136(2):448-456. 